\title{
Novas Fontes de Resistência do Feijoeiro Comum à Mancha Angular
}

\author{
Aloísio Sartorato \\ Embrapa Arroz e Feijão, Cx. Postal 179, CEP 75375-000, Santo Antônio de Goiás, GO, \\ email: sartorat@cnpaf.embrapa.br
}

(Aceito para Publicação em 12/12/2005)

Autor para correspondência: Aloísio Sartorato

SARTORATO, A. Novas fontes de resistência do feijoeiro comum à mancha angular. Fitopatologia Brasileira 31:192-194. 2006.

\section{RESUMO}

Vinte e oito genótipos, incluindo cultivares de algumas instituições de pesquisa e linhagens do programa de melhoramento da Embrapa Arroz e Feijão, foram avaliados quanto a resistência a oito patótipos do fungo Phaeoisariopsis griseola, agente causal da mancha angular do feijoeiro comum. Estes genótipos foram inoculados aos 14-16 dias após o plantio com uma suspensão contendo 2,0 x $10^{4}$ conídios $\mathrm{mL}^{-1}$. Os sintomas foram avaliados de 14 a 18 dias após a inoculação, utilizando uma escala de nove graus onde foram consideradas resistentes (reação compatível) as plantas que apresentaram os graus de 1 a 3 e suscetíveis (reação incompatível), as que apresentaram os graus de 4 a 9. Os genótipos ‘Ouro Negro’ e 'LM 202202530’ apresentaram reação de resistência a oito e a sete dos oito patótipos utilizados, respectivamente. A cultivar BRS Requinte foi resistente a cinco patótipos e as cultivares BRS Pontal e Cornell 49-242 foram resistentes a apenas quatro patótipos.

Palavras-chave adicionais: Phaeoisariopsis griseola, Phaseolus vulgaris, cultivares, patótipos.

\begin{abstract}
New sources of resistance to angular leaf spot on common bean

Twenty-eight bean genotypes and lines from the Embrapa Arroz e Feijão breeding program, and other research institutions, were evaluated for angular leaf spot reaction to eight pathotypes of Phaeoisariopsis griseola. Genotypes were inoculated 14-16 days after sowing by spraying an inoculum suspension containing $2.0 \times 10^{4}$ spores $\mathrm{mL}^{-1}$. Symptoms were evaluated 14-18 days after inoculation according to a 1-9 scale where disease ratings from 1 to 3 were considered resistant and 4 to 9 as susceptible. Genotypes 'Ouro Negro' and 'LM 202202530' were resistant to eight and seven pathotypes, respectively. Cultivar BRS Requinte was resistant to five pathotypes and cultivars BRS Pontal and Cornell 49-242 were resistant to only four pathotypes.
\end{abstract}

Additional keywords: Phaeoisariopsis griseola, Phaseolus vulgaris, cultivars, pathotypes.

A mancha angular do feijoeiro comum (Phaseolus vulgaris L.), cujo agente causal é o fungo Phaeoisariopsis griseola (Sacc.) Ferraris, é uma das principais doenças desta leguminosa. Segundo Dhingra \& Kushalappa (1980), P. griseola é transmitido pelas sementes em baixas porcentagens. Por esta razão, as sementes são pouco importantes na epidemiologia da doença. A mancha angular pode ser controlada através de práticas culturais, do uso de fungicidas e pela resistência genética do hospedeiro. As práticas culturais são pouco importantes. Atualmente, com o propósito de preservar tanto o ambiente como o homem, a sociedade vem solicitando à indústria e ao produtor um menor uso de pesticidas nas lavouras. Assim, a resistência genética do hospedeiro é, sem dúvida, o método mais eficiente, econômico e que menos risco oferece ao produtor, ao consumidor e ao ambiente. Entretanto, a obtenção de novas cultivares resistentes a esta enfermidade é dificultada pela variabilidade patogênica que $P$. griseola apresenta (Sartorato, 2002; Sartorato \& Alzate-Marin, 2004). O objetivo do presente estudo foi o de avaliar a resistência genética de 28 cultivares de feijoeiro comum a oito patótipos do fungo P. griseola em condições de casa de vegetação.

Foram utilizados os genótipos 'Magnífico', 'Carioca Rubi', Pérola, 'IAC Aruã', 'IAC Piatã', 'BRS Requinte', 'BRS Pontal', 'CNFC 09504', 'CNFC 10276', 'CNFC 10281', 'BRSMG Talismã', 'Uirapuru', 'Soberano', 'Diamante Negro', 'BRS Valente', 'BRS Grafite', 'CNFP 10120', 'CNFP 10138', 'Iapar 81', 'BRS Radiante', 'BRS Timbó', 'BRS Marfim', 'Ouro Negro', 'MAR 02', 'MAR 03’, 'CORNELL 49-242', 'LM 202202530' e o suscetível universal 'Rosinha G-2'. Estes genótipos foram semeados em vasos de alumínio (2,0 kg de solo), à razão de cinco sementes por genótipo. Os vasos contendo plantas de cada genótipo foram mantidos em casa de vegetação até o momento da inoculação. Na inoculação foram utilizados os seguintes patótipos, com a identificação dos respectivos isolados de $P$. griseola, entre parênteses, 31-31 (Ig 709), 63-15 (Ig 584.3), 63-23 (Ig 525.4), 63-31 (Ig 809), 63-47 
(Ig 874) 63-39 (Ig 60.4), 63-55 (Ig 784) e 63-63 (Ig 786). A produção do inóculo e a inoculação foram efetuados conforme descrito por Sartorato (2004). A avaliação dos sintomas foi realizada de 14 a 18 dias após a inoculação, utilizando uma escala de severidade de doença de nove graus (Sartorato \& Thung, 2002). Foram consideradas resistentes as plantas que apresentaram os graus de 1 a 3 (ausência de lesões com esporulação) e, suscetíveis, os graus de 4 a 9 (presença de lesões esporulantes) (Sartorato \& Thung, 2002). Quando as plantas inoculadas mostravam sintomas, mas não apresentavam esporulação do fungo na casa-de-vegetação, as mesmas foram transferidas para câmara úmida por 20-24 h. Após este período, as plantas que exibiam lesões não esporulantes foram consideradas resistentes (Sartorato, 2004). Para obtenção do dendrograma da Figura 1, foi utilizado o programa Statistica, versão 5.0 e os métodos Unweighted Pair-Group Average para análise de ligação e Squared Euclidean Distances na determinação da distância de ligamento.

Os resultados obtidos confirmam a grande dificuldade encontrada na identificação de genótipos com resistência ampla à mancha angular (Tabela 1). Dos 28 genótipos testados, 15 foram suscetíveis a todos os patótipos. Os testes de inoculação mostraram interações diferenciais entre alguns genótipos indicando resistência vertical (Nelson,
1978). Os genótipos 'Ouro Negro' e 'LM 202202530' apresentaram reação de resistência a oito e sete dos oito patótipos utilizados, respectivamente. A cultivar BRS Requinte foi resistente a cinco patótipos e as cultivares BRS Pontal e Cornell 49-242 a apenas quatro patótipos. A cultivar Ouro Negro, no presente estudo, apresentou reação de resistência completa aos patótipo 63-23 e 63-31. Entretanto, Ragagnin et al. (2003) encontraram neste genótipo uma mistura de reações apresentando plantas resistentes e suscetíveis aos referidos patótipos. Este fato pode indicar que a constituição genética desta cultivar não esteja em homozigose para a característica estudada. No presente estudo, foram observadas plantas desta cultivar com reações de resistência e de suscetibilidade aos patótipos 63-15, 6339, 63-47 e 63-63. A análise de agrupamento dos resultados obtidos dividiram os 28 genótipos em dois grandes grupos (Figura 1). No primeiro grupo estão incluídos os genótipos que apresentaram um maior grau de resistência à doenças (Ouro Negro, LM 202202530, BRS Grafite, BRS Pontal e RBS Requinte, Cornell 49-242 e MAR 03) e, no segundo grupo, os suscetíveis.

Em vista dos resultados obtidos, ou seja, considerando a presença de poucos genótipos com resistência a um grande número de patótipos, e a grande variabilidade que o fungo $P$. griseola apresenta, pode-se concluir que apenas a utilização

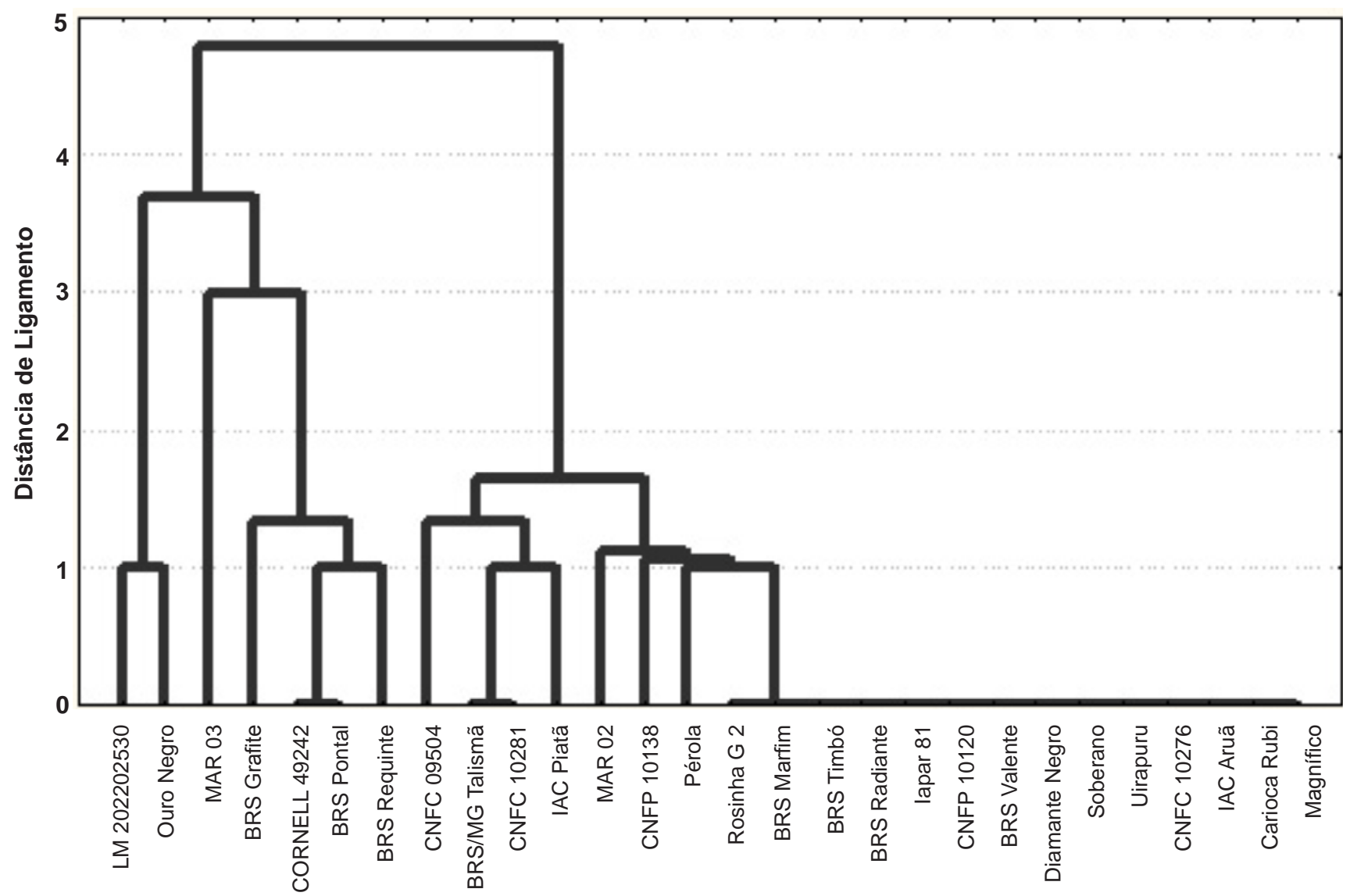

FIG. 1 - Dendrograma de 28 genótipos de feijoeiro comum (Phaseolus vulgaris) com base na inoculação de oito patótipos de Phaeoisariopsis griseola. 
TABELA 1 - Reação de 28 genótipos de feijoeiro comum (Phaseolus vulgaris) a oito patótipos de Phaeoisariopsis griseola

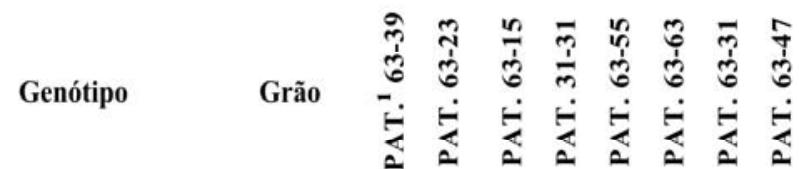

\begin{tabular}{|c|c|c|c|c|c|c|c|c|c|}
\hline Magnífico & Carioca & $\mathrm{S}^{2}$ & S & $S$ & $S$ & $\mathrm{~S}$ & $\mathrm{~S}$ & S & $\mathrm{S}$ \\
\hline Carioca Rubi & Carioca & S & S & S & S & S & S & S & $\mathrm{S}$ \\
\hline Pérola & Carioca & S & $\mathrm{R}$ & S & S & S & S & S & S \\
\hline IAC Aruã & Carioca & S & S & S & S & S & S & S & $\mathrm{S}$ \\
\hline IAC Piatã & Carioca & $\mathrm{S}$ & $\mathrm{R} / \mathrm{S}^{3}$ & S & S & $\mathrm{R} / \mathrm{S}$ & S & S & S \\
\hline BRS Requinte & Carioca & S & $\mathrm{R}$ & $\mathrm{R} / \mathrm{S}$ & $\mathrm{R} / \mathrm{S}$ & S & S & $\mathrm{R}$ & $\mathrm{R} / \mathrm{S}$ \\
\hline BRS Pontal & Carioca & S & $\mathrm{R}$ & $\mathrm{R}$ & $\mathrm{R} / \mathrm{S}$ & S & $\mathrm{S}$ & $\mathrm{R}$ & $\mathrm{S}$ \\
\hline CNFC 09504 & Carioca & S & S & S & $\mathrm{S}$ & $\mathrm{R} / \mathrm{S}$ & S & $\mathrm{R} / \mathrm{S}$ & S \\
\hline CNFC 10276 & Carioca & $\mathrm{S}$ & $\mathrm{S}$ & $\mathrm{S}$ & $\mathrm{S}$ & S & $\mathrm{S}$ & $\mathrm{S}$ & $\mathrm{S}$ \\
\hline CNFC 10281 & Carioca & $\mathrm{S}$ & $\mathrm{S}$ & $\mathrm{S}$ & $\mathrm{S}$ & S & $\mathrm{S}$ & $\mathrm{S}$ & $\mathrm{S}$ \\
\hline BRS/MG Talismã & Carioca & S & S & S & S & $\mathrm{R}$ & S & S & S \\
\hline Uirapuru & Preto & S & S & S & S & S & S & S & S \\
\hline Soberano & Preto & S & S & S & S & S & $\mathrm{S}$ & S & S \\
\hline Dia Negro & Preto & S & S & S & S & S & S & S & S \\
\hline BRS Valente & Preto & S & S & S & S & S & S & S & $\mathrm{S}$ \\
\hline BRS Grafite & Preto & S & $\mathrm{R}$ & $\mathrm{S}$ & $\mathrm{R} / \mathrm{S}$ & S & S & $\mathrm{R} / \mathrm{S}$ & $\mathrm{S}$ \\
\hline CNFP 10120 & Preto & S & S & S & S & S & S & S & $\mathrm{S}$ \\
\hline CNFP 10138 & Preto & S & S & $\mathrm{R}$ & S & S & $\mathrm{S}$ & S & $\mathrm{S}$ \\
\hline Iapar 81 & Preto & S & S & S & S & S & S & S & $\mathrm{S}$ \\
\hline BRS Radiante & Rajado & S & S & S & S & S & S & S & S \\
\hline BRS Timbó & Roxinho & $\mathrm{S}$ & $\mathrm{S}$ & S & $\mathrm{S}$ & $\mathrm{S}$ & $\mathrm{S}$ & S & $\mathrm{S}$ \\
\hline BRS Marfim & Mulatinho & S & S & S & S & S & S & S & $\mathrm{S}$ \\
\hline Ouro Negro & Preto & $\mathrm{R} / \mathrm{S}$ & $\mathrm{R}$ & $\mathrm{R} / \mathrm{S}$ & $\mathrm{R}$ & $\mathrm{R} \quad \mathrm{I}$ & $\mathrm{R} / \mathrm{S}$ & $\mathrm{R}$ & $\mathrm{R} / \mathrm{S}$ \\
\hline MAR 02 & Mulatinho & $\mathrm{R}$ & S & S & S & S & $\mathrm{S}$ & S & $\mathrm{S}$ \\
\hline MAR 03 & Carioca & $\mathrm{R}$ & $\mathrm{R}$ & $\mathrm{S}$ & $\mathrm{R}$ & S & $\mathrm{S}$ & $\mathrm{S}$ & $\mathrm{S}$ \\
\hline CORNELL 49242 & Preto & S & $\mathrm{R} / \mathrm{S}$ & $\mathrm{R} / \mathrm{S}$ & $\mathrm{R}$ & S & $\mathrm{S}$ & $\mathrm{R}$ & S \\
\hline LM 202202530 & Preto & $\mathrm{R} / \mathrm{S}$ & $\mathrm{R} / \mathrm{S}$ & $\mathrm{R} / \mathrm{S}$ & $\mathrm{R} / \mathrm{S}$ & $\mathrm{R} / \mathrm{S}$ & S & $\mathrm{R} / \mathrm{S}$ & $\mathrm{R} / \mathrm{S}$ \\
\hline Rosinha G 2 & Rosinha & S & S & S & $\mathrm{S}$ & S & $\mathrm{S}$ & S & S \\
\hline
\end{tabular}

da resistência vertical completa não é a melhor estratégia a ser utilizada no controle desta doença. Como resultado, sugere-se que sejam também utilizados, sempre que possível, como fonte de resistência, genótipos que apresentem os mais altos graus de resistência horizontal e parcial (Sartorato \& Rava, 1993). Uma outra possibilidade para o controle da mancha angular do feijoeiro comum é o desenvolvimento e utilização de cultivares multilinhas.

O patótipo 63-63 foi o mais virulento entre os oitos empregados no estudo. O genótipo Ouro Negro foi o único a apresentar plantas com reação de resistência a este patótipo. Até recentemente, este patótipo apresentava virulência a todos os genótipos de feijoeiro comum testados. Consequentemente, recomenda-se a utilização dos genótipos Ouro Negro ou LM 202202530 (que apresenta na sua genealogia a cultivar Ouro Negro), em programas de melhoramento que visem a resistência do feijoeiro comum a mancha angular.

\section{REFERÊNCIAS BIBLIOGRÁFICAS}

DHINGRA, O.D. \& KUSHALAPPA, A.C. No correlation between angular leaf spot intensity and seed infection in bean by I. griseola. Fitopatologia Brasileira 5:149-152. 1980.

NELSON, R.R. Genetics of horizontal resistance to plant diseases. Annual Review of Phytopathology 16:359-378. 1978.

RAGAGNIN, V.A., ALZATE-MARIN, A.L., SOUZA, T.L.P.O., ARRUDA, K.M.A., MOREIRA, M.A. \& BARROS, E.G. Avaliação da resistência de isolinhas de feijoeiro a diferentes patótipos de Colletotrichum lindemuthianum, Uromyces appendiculatus e Phaeoisariopsis griseola. Fitopatologia Brasileira 28:591-596. 2003.

SARTORATO, A. Identification of Phaeoisariopsis griseola pathotypes from five States in Brazil. Fitopatologia Brasileira 27:78-81. 2002.

SARTORATO, A. Pathogenic variability and genetic diversity of Phaeoisariopsis griseola isolates from two counties in the State of Goiás, Brazil. Journal of Phytopathology 152:385-390. 2004.

SARTORATO, A. \& RAVA, C.A. Determinação da resistência parcial do feijoeiro comum a Isariopsis griseola. Anais, $4^{\mathrm{a}}$ Reunião Nacional de Pesquisa de Feijão. Londrina PR. 1993.

SARTORATO, A. \& ALZATE-MARIN, A.L. Analysis of the pathogenic variability of Phaeoisariopsis griseola in Brazil. Annual Report of the Bean Improvement Cooperative 447:235-236. 2004.

SARTORATO, A. \& THUNG, M. Determinação da variabilidade patogênica de Phaeoisariopsis griseola e avaliação da mancha angular. In: Sartorato, A. \& Thung, M. (Eds.) Memórias da participação brasileira no "I Taller Internacional sobre la Mancha Angular del Frijol”, Santo Antônio de Goiás, GO. 2002. Disponível em:http://www.cnpaf.embrapa.br/publicacao/seriedocumentos/doc 132/132 4.htm 